\title{
A multimeasure approach to investigating affective appraisal of social information in Williams syndrome
}

\author{
Daniela Plesa Skwerer • Emily Ammerman • \\ Marie-Christine André • Lucia Ciciolla • Alex B. Fine • \\ Helen Tager-Flusberg
}

Received: 13 May 2011 / Accepted: 31 October 2011 / Published online: 12 November 2011

(C) Springer Science+Business Media, LLC 2011

\begin{abstract}
People with Williams syndrome (WS) have been consistently described as showing heightened sociability, gregariousness, and interest in people, in conjunction with an uneven cognitive profile and mild to moderate intellectual or learning disability. To explore the mechanisms underlying this unusual social-behavioral phenotype, we investigated whether individuals with WS show an atypical appraisal style and autonomic responsiveness to emotionally laden images with social or nonsocial content. Adolescents and adults with WS were compared to chronological age-matched and nonverbal mental agematched groups in their responses to positive and negative images with or without social content, using measures of self-selected viewing time (SSVT), autonomic arousal reflected in pupil dilation measures, and likeability ratings. The participants with WS looked significantly longer at the social images compared to images without social content and had reduced arousal to the negative social images compared to the control groups. In contrast to the
\end{abstract}

D. Plesa Skwerer $(\bowtie) \cdot$ E. Ammerman $\cdot$ H. Tager-Flusberg Department of Psychology, Boston University,

64 Cummington Street \#126C,

Boston, MA 02115, USA

e-mail: dplesas@bu.edu

M.-C. André

Psychology Department, Suffolk University,

Boston, MA, USA

L. Ciciolla

Department of Psychology, Arizona State University,

Tempe, AZ, USA

\section{A. B. Fine}

Department of Brain and Cognitive Science, University of Rochester,

New York, NY, USA comparison groups, the explicit ratings of likeability in the WS group did not correlate with their SSVT; instead, they reflected an appraisal style of more extreme ratings. This distinctive pattern of viewing interest, likeability ratings, and autonomic arousal to images with social content in the WS group suggests that their heightened social drive may be related to atypical functioning of reward-related brain systems reflected in SSVT and autonomic reactivity measures, but not in explicit ratings.

Keywords Williams syndrome A Autonomic reactivity Social phenotype $\cdot$ Appraisal style

Williams syndrome (WS) is a neurodevelopmental disorder caused by a microdeletion of about 25 genes in the $7 \mathrm{q} 11.23$ region of chromosome 7 (Ewart et al. 1993; Korenberg et al. 2000). It is associated with mild to moderate intellectual disabilities and an unusual cognitive and social phenotype. Among some of the striking features of the socialbehavioral profile of individuals with WS is their heightened attention to human faces in real-life situations, empathy, and social disinhibition, coexisting with generally elevated anxiety. People with WS are described as overly friendly and eager to approach and interact with other people, including strangers, but also as having difficulties in forming and maintaining friendships and sometimes experiencing social isolation by adulthood (Doyle et al. 2004; Gosch and Pankau 1997; Howlin and Udwin 2006; Jones et al. 2000; Plesa Skwerer et al. 2004; Stinton et al. 2010). Although there is substantial agreement in the field regarding the "hypersociability" of individuals with WS, the neurocognitive mechanisms underlying this striking profile have remained elusive. To date, most experimental studies tapping the social phenotype of WS have focused on explicit responses to stimuli, such as accuracy in face 
recognition (Bellugi et al. 1994; Rose et al. 2007; TagerFlusberg et al. 2003), identifying emotional expressions (Gagliardi et al. 2003; Plesa Skwerer et al. 2006a, b; Porter et al. 2007), and direction of gaze (Mobbs et al. 2004) or performance on tasks probing social cognition (John and Mervis 2010; Porter et al. 2008; Tager-Flusberg and Sullivan 2000).

Several studies have used functional magnetic resonance imaging (fMRI) to examine the neural mechanisms underlying the atypical social profile in WS. These studies focused on the amygdala and its regulatory links to other cortical regions implicated in the processing of socially relevant information and in monitoring social behavior (Haas et al. 2009; Meyer-Lindenberg et al. 2005; MeyerLindenberg et al. 2006; Paul et al. 2009). More specifically, researchers have hypothesized that inadequate interpretation of social danger signals in the environment might explain the increased affiliative drive and social approach behavior seen in WS, linked to functional abnormalities in the amygdala (Bellugi et al. 1999; Meyer-Lindenberg et al. 2005; Muñoz et al. 2010; Paul et al. 2009; Porter et al. 2007). Meyer-Lindenberg et al. (2005) found significant hypoactivation of the amygdala in response to negative facial affect in WS individuals with average IQ compared to age-matched controls, but abnormally increased amygdala activation in WS when presented with threatening and fearful scenes without social content. Path analyses of the activation patterns in the two groups showed altered amygdala-prefrontal connectivity in WS, suggesting that abnormalities in the regulatory interactions between the prefrontal cortex and the amygdala could explain the pattern of decreased social fear and increased nonsocial fear reported in people with WS (Meyer-Lindenberg et al. 2005; Muñoz et al. 2010). This interpretation of decreased responsiveness to or recognition of social threat in WS has been supported by other types of evidence showing autonomic hypoarousal to negative facial expressions (Plesa Skwerer et al. 2009) or unusually high ratings of unfamiliar faces on approachability and trustworthiness dimensions (Bellugi et al. 1999; Martens et al. 2009; Porter et al. 2007). Plesa Skwerer et al. (2009) examined autonomic arousal in response to dynamically presented facial expressions of emotion in adolescents and adults with WS, compared to age-matched normal control participants and to age-, IQ-, and language-matched individuals with learning or intellectual disabilities (LID group), using skin conductance responses and heart rate as amygdala-mediated measures of autonomic responsiveness (cf. Adolphs 2001; LeDoux 2000). Compared to both control groups, the WS participants showed reduced skin conductance amplitudes, indicating hypoarousal to the socially relevant stimuli, and greater heart rate deceleration, suggesting heightened interest in the dynamically presented facial expressions. In contrast to these autonomic reactivity differences, the WS group did not differ from the LID group in their ability to label the facial emotional expressions, though, as in several other studies (Gagliardi et al. 2003; Plesa Skwerer et al. 2006a, b; Porter et al. 2007), their performance on this explicit task was impaired in comparison to normal controls.

Another task that has been widely used to probe social judgments of other individuals on the basis of their facial appearance and that involves amygdala activation was developed by Adolphs et al. (1998). In this task, participants are asked to rate 100 faces of unfamiliar people with respect to two attributes important in real-life social encounters: approachability and trustworthiness. Adolphs et al. (1998) found that individuals with bilateral amygdala damage usually provided higher ratings than controls on the two dimensions when using facial appearance for their social judgments, but not when judging verbal descriptions of people. Several studies have investigated ratings of approachability and trustworthiness of faces using the Adolphs task or a variation of this task (Frigerio et al. 2006; Porter et al. 2007) in WS and reported that individuals with WS provided higher positive ratings to faces than control groups, although Frigerio et al. (2006) found this to be true only for faces with happy expressions.

To date, most studies that aimed to examine the underlying mechanisms of the hypersocial behavior seen in WS used individual faces as target images in various tasks probing either explicit or implicit responses to social stimuli. However, none of these studies employed multiple types of measures that could disentangle the contributions of attention, autonomic arousal, and conscious interest in processing socially relevant information. Our goal was to expand this line of research by including more complex socially relevant scenes instead of isolated faces and to include measures that would enable us to distinguish explicit appraisal from responses to the stimuli reflected in viewing time as an implicit index of interest and in autonomic arousal as a psychophysiological index of sympathetic reactivity to social-emotional stimuli. We were particularly interested in exploring whether images portraying people in complex scenes suggesting positive or negative experiences (e.g., children playing in an amusement park, women crying at a funeral) would elicit different attention deployment and autonomic responses from WS participants than images without social content.

To assess the differential appraisal of emotionally laden images with social or nonsocial content, we employed a set of measures probing both explicit and implicit processing of the visual information, including measures of selfselected viewing time (SSVT), changes in pupil size during stimulus viewing, and ratings of likeability. We hypothesized that the SSVT and autonomic responses in the WS 
group would differ from those of control groups of typically developing individuals matched on chronological age (CA) or on nonverbal mental age (MA), primarily as a function of the social or nonsocial content of the stimulus images. Specifically, we expected that the WS participants would look longer at social images compared to images with nonsocial content and compared to the SSVT of both control groups for social images, that they would rate social images as more likable than nonsocial images of the same valence, and that they would provide higher ratings than control groups for the positive social images. We also hypothesized that the WS group would show smaller pupil-dilation differences compared to both control groups on images with negative social content, consistent with the decreased autonomic responsiveness to negatively valenced social stimuli shown by individuals with WS in other studies.

\section{Methods}

\section{Participants}

This study included 42 (21 females) adolescents and young adults with WS, matched on CA $(p=0.25)$ with 40 (24 females) typical control participants (CA group) and on nonverbal MA $(p=0.98)$ with 37 (20 females) typically developing children (MA group). All participants were administered the Kaufman Brief Intelligence Test, second edition (KBIT-2; Kaufman and Kaufman 2004) as a general cognitive assessment that enabled us to match participants on nonverbal MA. Table 1 presents details of the participant groups. Because not all participants had complete, valid data on all measures, the composition of each group differed slightly for each measure, but the group matching on age or nonverbal MA did not differ with the smaller groups. All the WS participants exhibited the medical and clinical features of the WS phenotype (Bellugi et al. 1994; Mervis et al. 2000; Morris et al. 1988) and the diagnosis of WS was confirmed on genetic testing (FISH test). Participants were screened for psychiatric and/or neurological problems and none was taking any psychotropic medications at the time of the study.
Stimulus preparation and piloting

The final set of stimuli consisted of 60 images, of which 41 were from the International Affective Picture System (IAPS; Lang et al. 2008) and the rest were from Internet sources, selected based on the content and emotional valence conveyed by the image. Before selecting the final set of stimuli, we conducted a preliminary validation of an initial larger collection of 70 images which included some images from other sources than the IAPS, with a group of typical young adult judges (instead of relying on published normative ratings for IAPS), to ensure that the pictures conveyed the intended emotional valence. The initial larger set of images was rated by 12 adult judges who used a 7-point Likert scale (from -3 to +3 ) to express the degree of unpleasant to pleasant emotional valence conveyed by each image. Any image that received discrepant ratings differing by more than 3 points from at least three raters was excluded from the final set. This was done to assure consistency in the categorical aspect of emotional appraisal (i.e., positive/negative valence) as judged by typical adults. The remaining pictures received ratings across the range within the appropriate valence (e.g., positive images were rated from 1 to 3 ). The images in this set, subtending a visual angle of $18^{\circ}$ vertically and $12-23^{\circ}$ horizontally, were then checked for luminance levels using Adobe Photoshop 7.0.1 to ensure consistency in mean luminance across categories of stimuli, and no significant differences in mean luminance between categories of images were found. The final set of stimuli included 60 images that were equally divided between 4 categories (15 images in each category): social-positive (e.g., smiling people, mother and baby playing), nonsocial-positive (e.g., flowers, gift box), social-negative (e.g., injured people, women crying at a funeral), and nonsocial-negative (e.g., a gun, house on fire).

\section{Procedure}

\section{Self-selected viewing time and pupillometry}

Participants were seated in front of a computer screen at a distance of approximately $60 \mathrm{~cm}$ from the monitor and were told that they would be shown different images, some of

Table 1 Participant characteristics

\begin{tabular}{|c|c|c|c|c|c|c|c|c|c|}
\hline & \multicolumn{3}{|c|}{ WS $(n=42)$} & \multicolumn{3}{|c|}{ CA match $(n=40)$} & \multicolumn{3}{|c|}{ MA match $(n=37)$} \\
\hline & $M$ & SD & Range & $M$ & SD & Range & $M$ & SD & Range \\
\hline $\mathrm{CA}$ & 22.19 & 5.69 & $12.5-34.5$ & 20.64 & 6.33 & $12.7-34.6$ & 7.94 & 1.81 & $5.1-11.3$ \\
\hline Full-scale IQ (KBIT-2) & 68.8 & 12.4 & $41-91$ & 111.2 & 10.6 & $90-127$ & 106.0 & 10.7 & $86-126$ \\
\hline Nonverbal MA (KBIT-2) & 8.18 & 2.69 & $4.5-14.7$ & 16.87 & 2.39 & $10.3-18.5$ & 8.20 & 3.03 & $4.0-14.7$ \\
\hline
\end{tabular}

$\mathrm{CA}$ and MA are reported in years 
which they might like and others they might dislike. The images were displayed on a TOBII-1750 monitor and their presentation was programmed using Eprime 1.1 experimental software. The order of the images was pseudorandomized, with the constraint that no more than three images from the same category were presented in a row.

The TOBII-1750 eye tracker recorded points of regard coordinates and pupil diameter approximately every $20 \mathrm{~ms}$. A button box, connected to a DELL Latitude D610 computer running the stimulus presentation script, was used to advance from one image to the next, providing response-time information. Participants were instructed that pressing one button on the button box would advance to the next image, but that the experiment lasted for a fixed time $(6 \mathrm{~min})$. The experimenter emphasized that, by pushing the "next" button, they could control the viewing time of each image, but not the length of the experiment; therefore, they could spend more time looking at images they liked because the pictures would start repeating if they went too quickly. To familiarize the participants with the use of the button box, there was a practice phase before the presentation of the experimental stimuli. During the practice phase, six images (different from the test stimuli), some extremely pleasant or unpleasant, were displayed one at a time, and the experimenter explained that the image presentation was untimed, so the subject had to push the button to advance. After the practice trials, the 60 test images were presented in a loop for $6 \mathrm{~min}$. In this paradigm, the total viewing time for each stimulus was taken as an index of interest in the image.

Pupil size recordings provided every $20 \mathrm{~ms}$ by the eye tracker were used to calculate stimulus-related changes in pupil size. Dilation in pupil size is generally considered to indicate an autonomic response to the perceived emotional undertone of visual information, most commonly associated with threatrelated images connoting fear or aversion (Granholm and Steinhauer 2004; Hess 1972; Janisse 1973; Libby et al. 1973). Pupil dilation may also be a response to extreme interest in a stimulus of significant personal relevance (e.g., one's own child), connoting heightened pleasure, or to stimuli with high arousal value (e.g., erotica). Because our samples included a group of young children (MA) and one of individuals with intellectual disabilities (WS), we excluded the latter category, as well as all images of animals (to avoid any ambiguity about the social/nonsocial content of such images), and none of the positively valenced images were expected to have high personal relevance for any participants. Therefore, we focused the analyses in this study on pupil size changes only to the negative images.

\section{Image ratings}

After participants had completed the computer task, they were given a booklet containing the same images viewed on the computer monitor, but displayed in a different random order, with eight images per page. Participants were instructed to rate how much they liked each of the 60 images on a 7-point Likert scale, from $-3=$ strongly dislike to $+3=$ strongly like. A line representation of the scale was printed below each image in the booklet. To avoid any confusions about the meaning of negative numbers, the experimenter first asked the young participants in the MA group and any participant who seemed to struggle with the 7-point scale to report their ratings orally, first saying whether they liked or disliked the picture, and then guiding them through the process of selecting a corresponding number for how much they liked or disliked it.

\section{Results}

\section{Self-selected viewing time}

Participants who looped through all 60 images more than 4 times were excluded from the SSVT analyses because their button-box behavior clearly indicated that they ignored the content of the images, as they most likely spent too little viewing time per image $(<1.3 \mathrm{~s})$ to process anything beyond the most superficial visual features of the complex images. There were $4 \mathrm{WS}, 1 \mathrm{CA}$, and $5 \mathrm{MA}$ participants who were excluded, leaving $38 \mathrm{WS}, 39 \mathrm{CA}$, and $32 \mathrm{MA}$ participants included in the viewing time analyses. In these groups, there were 13 individuals with WS, 13 CA participants, and 8 MA participants who did not finish going through all 60 images in the allotted experimental time, but all had seen at least 3 images from each category and, therefore, their SSVT data were included in analyses.

The measure of viewing time used in the statistical analyses was derived in the following way. We calculated the mean "baseline" viewing time (in milliseconds) for each participant as the mean of each individual response time across the entire experiment. Subtracting this "baseline" viewing time measure from the mean SSVT for each of the four categories targeted provided a measure of greater interest - indexed by positive differences for the four categories of images or reduced interest (or avoidance) indexed by negative differences for the four categories of images.

The data for each group are presented in Table 2. A mixed-model ANOVA conducted on the viewing time difference scores, using image content (social, nonsocial) and valence (positive, negative) as within-subjects factors and group (WS, CA, MA) and sex as between-subjects factors, yielded a significant main effect of valence, $F$ (1, $103)=11.19, p=0.001, \eta_{\mathrm{p}}{ }^{2}=0.098$, and a significant interaction between image content and group, $F(2,103)=4.28$, $p=0.016, \eta_{\mathrm{p}}{ }^{2}=0.077$. There were no other significant main 
Table 2 SSVT (difference from baseline viewing time in milliseconds) by group and image category

\begin{tabular}{lllrr}
\hline & & WS & \multicolumn{1}{l}{ CA match } & \multicolumn{1}{l}{ MA match } \\
& & M (SD) & M (SD) & M (SD) \\
\hline Positive & Social & $1,657(4,369)$ & $457(1,224)$ & $519)$ \\
& Nonsocial & $648(2,333)$ & $542(2,151)$ & $1,150(2,318)$ \\
Negative & Social & $-1,096(4,354)$ & $-407(1,652)$ & $-361(1,831)$ \\
& Nonsocial & $-1,667(3,534)$ & $-468(1,559)$ & $-602(1,556)$ \\
& & & &
\end{tabular}

effects or interactions. In all groups, participants looked longer at positive $(M=663.6 \mathrm{~ms})$ than negative $(M=-808.9 \mathrm{~ms})$ images. The interaction between content and group was further analyzed by comparing viewing time difference scores to social and nonsocial images in each group, as well as by comparing the groups on viewing time difference scores separately for social and nonsocial images, using Bonferroni adjustments for multiple comparisons. Post hoc comparisons within groups indicated that the WS participants looked longer at social than nonsocial images $(p=0.011)$, while neither comparison group showed a content-related difference in their looking time relative to their baseline. Post hoc comparisons between groups within image content categories indicated that the WS group looked significantly less at nonsocial images than the MA group relative to their baseline looking time $(p=0.008)$ and showed a similar trend when compared to the CA group $(p=0.082)$, while the MA and CA groups did not differ from each other $(p=0.96)$.

\section{Pupil-change scores}

Each subject's baseline pupil size (in millimeters) was obtained as the mean of pupil diameter recordings across all the images presented during the 6-min experiment. Then, for each image, we computed change scores represented by the differences between pupil diameter for that image and the participant's baseline, after discarding the pupil data of the respective image targeted from its baseline calculation. Then, means of these change scores were computed for each of the four categories of images. The measurement of pupil size for each stimulus was made starting at $500 \mathrm{~ms}$ after the onset of the stimulus and ending either after $3 \mathrm{~s}$ of viewing or when the image display was changed by the participant, whichever came first. Pupil size measurements were used to create pupil-change scores only if a minimum of $200 \mathrm{~ms}$ of actual viewing time for each image was recorded as valid data (by the eye tracker). Only participants who had valid data for at least 3 of the 15 images in each category were included in these analyses (to be consistent with the inclusion criterion used for the SSVT). Based on these data screening criteria, $30 \mathrm{WS}, 30 \mathrm{CA}$, and 32 MA participants were included in the statistical analyses.
Figure 1 presents the data for each group of pupil-change means from baseline by image content.

A mixed-model ANOVA was conducted on mean pupilchange scores for the negative images with image content (social, nonsocial) as a within-subjects factor and group (WS, CA, MA) and sex as between-subjects factors. This analysis yielded a significant main effect of content, $F$ (1, $86)=103.40, p<0.001, \eta_{\mathrm{p}}{ }^{2}=0.546$; group, $F(2,86)=5.46$, $p=0.006, \eta_{\mathrm{p}}{ }^{2}=0.113 ;$ a significant content by group interaction, $F(2,86)=3.49, p=0.035, \eta_{\mathrm{p}}{ }^{2}=0.075$; and a significant content by sex interaction, $F(1,86)=6.42, p=$ $0.013, \eta_{\mathrm{p}}{ }^{2}=0.069$. Across groups, pupil-change scores were significantly higher for social-negative $(M=0.10)$ than nonsocial-negative images $(M=-0.014)$. Across all negative images, pupil-change scores were significantly higher for the CA group than for the WS $(p=0.023)$ and the MA $(p=0.01)$ groups, which did not differ from each other.

The significant interaction between content and group was further analyzed by examining the pattern of group differences separately for images with social and nonsocial content. Within social-negative images, pupil-change scores were significantly lower for the WS group than the CA group $(p=0.004)$, but the WS and MA groups did not differ from each other. For the nonsocial content images, there were no significant differences in the pupil-change measure between the WS and either control group, although the CA group showed, on average, higher pupil dilation than the MA group $(p=0.05)$. Follow-up analyses of the significant

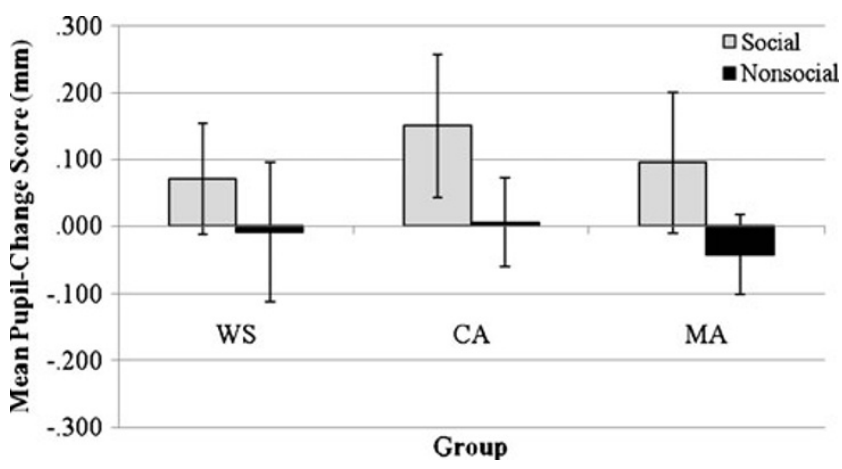

Fig. 1 Mean pupil-change scores (in millimeters) for negative images by group and image content. Error bars represent standard deviations 
interaction between sex and image content indicated that, within social-negative images across groups, pupil-change scores were significantly higher for females than for males $(p=0.016)$, but there were no differences on the nonsocial images.

\section{Image ratings}

Participants whose mean ratings were outliers in the opposite direction from their group mean within-image category (one in the WS, two in the CA, and three in the MA group) or who provided incomplete data on the likeability ratings measure (five WS, four $\mathrm{CA}$, and one MA) were excluded from analyses. Based on these data screening criteria, $35 \mathrm{WS}, 34 \mathrm{CA}$, and $33 \mathrm{MA}$ participants were included in the statistical analyses for subjective ratings. Analyses were conducted separately for positively valenced and negatively valenced images.

\section{Positive valence}

A mixed-model ANOVA was conducted on mean likeability ratings with image content (social-positive, nonsocial-positive) as a within-subjects factor and group (WS, CA, MA) and sex as between-subjects factors. This analysis yielded significant main effects of content, $F(1$, 96) $=25.56, p<0.001, \eta_{\mathrm{p}}{ }^{2}=0.21$; group, $F(2,96)=7.92, p=$ $0.001, \eta_{\mathrm{p}}{ }^{2}=0.142$; and sex, $F(1,96)=11.21, p=0.001$, $\eta_{\mathrm{p}}{ }^{2}=0.105$; and a significant group by content interaction, $F(2,96)=5.54, p=0.005, \eta_{\mathrm{p}}{ }^{2}=0.103$. Overall, participants rated nonsocial images $(M=1.93)$ higher than social images $(M=1.52)$, females gave higher ratings than males ( $M=1.97$ and $M=1.49$, respectively), and the WS participants gave higher ratings than either the CA $(p<0.001)$ or the MA $(p=0.012)$ participants, who did not differ from each other (see Table 3).

Post hoc comparisons following up on the significant group by content interaction indicated that both comparison groups rated nonsocial images significantly higher than social images (CA: $p=0.049$; MA: $p<0.001$ ), but there was no significant difference for the WS participants. The WS group gave higher ratings to social images than both the CA group $(p=0.005)$ and the MA group $(p=0.001)$ and also

Table 3 Mean likeability rating by group and image category

\begin{tabular}{lllll}
\hline & & WS & \multicolumn{1}{l}{ CA match } & \multicolumn{1}{l}{$\begin{array}{l}\text { MA match } \\
\text { M (SD) }\end{array}$} \\
\hline Positive & Social & $2.04(0.66)$ & $1.39(0.63)$ & $1.23(1.39)$ \\
& Nonsocial & $2.20(0.66)$ & $1.67(0.78)$ & $2.00(0.78)$ \\
Negative & Social & $-1.77(0.82)$ & $-1.24(1.04)$ & $-1.17(1.27)$ \\
& Nonsocial & $-2.04(0.80)$ & $-1.48(1.03)$ & $-1.17(1.25)$ \\
\hline
\end{tabular}

gave higher ratings to nonsocial images than the CA group $(p=0.003)$. The two control groups did not differ significantly from one another for either image category.

\section{Negative valence}

A mixed-model ANOVA was conducted on mean likeability ratings with image content (social-negative, nonsocial-negative) as a within-subjects factor and group (WS, CA, MA) and sex as between-subjects factors. This analysis yielded significant main effects of content, $F(1$, 96) $=4.73, p=0.032, \eta_{\mathrm{p}}{ }^{2}=0.047$; group, $F(2,96)=6.83$, $p=0.002, \eta_{\mathrm{p}}{ }^{2}=0.125$; and sex, $F(1,96)=16.10, p<0.001$, $\eta_{\mathrm{p}}{ }^{2}=0.144$, with no significant interactions. Overall, participants rated nonsocial images $(M=-1.51)$ more negatively than social images $(M=-1.35)$, females gave more negative ratings than males $(M=-1.8$ and $M=-1.07$, respectively), and the WS participants gave more negative ratings than either the $\mathrm{CA}(p=0.011)$ or the $\mathrm{MA}(p=0.003)$ groups, who did not differ from each other.

\section{Correlations between measures}

Pupil-dilation differences were not related to the other measures (SSVT, ratings) for any of the groups. Table 4 summarizes the correlations between viewing time and likeability ratings for each group. In the WS group, no systematic relationships were found between viewing time and ratings. In contrast, in the CA and MA comparison groups, significant correlations were found between viewing time and likeability ratings for all image categories, with the exception of nonsocial-positive and social-negative images in the MA group.

We also conducted analyses of relations between the three experimental measures and age and cognitive functioning (IQ scores) and found no correlations with age in the WS group for any measures. However, IQ and nonverbal MA were correlated only to the "baseline" viewing time in WS, suggesting that, in general, higher cognitive functioning participants with WS tended to spend more time viewing the images, whereas lower-functioning individuals with WS tended to rush through the images.

Table 4 Correlations between likeability ratings and SSVT differences from baseline

\begin{tabular}{lllll}
\hline & & WS & CA match & MA match \\
\hline Positive & Social & 0.046 & $0.384^{*}$ & $0.403^{*}$ \\
& Nonsocial & 0.012 & $0.517^{* *}$ & 0.164 \\
Negative & Social & 0.164 & $0.421^{*}$ & 0.325 \\
& Nonsocial & 0.052 & $0.527^{* *}$ & $0.548^{* *}$ \\
\hline
\end{tabular}

$* p<0.05, * * p<0.01 ;$ Spearman's rho 
However, the viewing time difference scores calculated as a measure of interest/disinterest in categories of images relative to baseline viewing duration were not related to age in the WS group. There were no other significant correlations between study measures and age/cognitive scores in the WS group.

\section{Discussion}

The goal of this study was to conduct an experimental investigation of a distinctive component of the social phenotype of WS - appraisal of emotional stimuli-using a multimeasure approach. Each of the measures yielded interesting distinctive patterns of responding by the WS participants in comparison to the typically developing age or MA controls. First, the WS group looked relatively longer at the social images on our novel SSVT measure. Second, compared to the CA group (though not the MAmatched group), the WS participants showed less pupil dilation in response to negative social images, suggesting reduced responsiveness to social threat relative to normal controls. Third, the ratings of the WS participants were more extreme in both the positive and negative directions than either comparison group. And, finally, in contrast to the other groups, viewing time was unrelated to the ratings of the images by the participants with WS. In this discussion, we take up each of these findings and discuss the implications for what makes people with WS so distinctive in their social interactions with others.

The stimuli we selected for this study were more diverse and complex than standard face-processing tasks. The complex social and nonsocial scenes that varied in their emotional impact were especially well suited to the selfselective viewing task in which participants were able to control the amount of time and hence attention that was allocated to a large number of images. Our finding that the WS participants spent significantly longer viewing time on both positive and negative social images was consistent with our predictions. A key facet of the WS phenotype is their especially strong interest in people. Developmental studies suggest that this is a very early emerging aspect of the WS phenotype, evident in infants and toddlers (Jones et al. 2000; Mervis et al. 2003). Studies of older children with WS used eye-tracking measures and found that they spent longer looking at faces than comparison groups (e.g., Riby and Hancock 2008, 2009). One study by Dodd and Porter (2010) used a dot-probe task with pairs of faces exhibiting different emotional expressions. On this task, attention to faces was only greater in the WS group for the happy faces, not to the faces exhibiting negative or neutral expressions. This finding was interpreted as showing that the WS group was reluctant to disengage from the happy faces rather than pure attention capture. In our study, which used a different measure of attention, longer viewing time among the participants with WS was found for both positive and negative social scenes, and therefore, suggests that, relative to nonsocial scenes, adolescents and adults with WS show a distinctive pattern of choosing to spend more time looking at any image that included people.

As the participants looked at the images presented, arousal to the negative images was assessed using a pupil dilation measure indexed to each participant's baseline pupil size. The social scenes were more arousing to all the participants in the study compared to the nonsocial scenes, though the WS group showed less increase in pupil size than the typical controls. This difference was significant in comparison to the CA matches, but not the MA matches. However, given the developmental changes in the shape and the activity of the pupil (e.g., pupil diameters decrease with age, the relationship between pupil responsiveness and initial pupil diameter changes with age-see Lowenfeld 1979; Kasthurirangan and Glasser 2006), it may be that our pupil dilation measure is not comparable across different age groups, suggesting that, at least for this measure, the CA group is more appropriate for comparison to the WS group. The lower pupil dilation among the WS participants suggests that they did not find the social-negative images as arousing as their age-matched controls, a finding that is consistent with other studies that have used a variety of different psychophysiological measures of autonomic arousal. For example, Plesa Skwerer et al. (2009) found that, in comparison to well-matched controls, adolescents and adults with WS showed less elevated skin conductance responses and heart rate deceleration, indicating interest rather than arousal to dynamic faces expressing a range of emotions. Functional imaging studies also have found less amygdala activation when WS participants were shown threatening faces (Haas et al. 2009; Meyer-Lindenberg et al. 2005). Together, these findings confirm that people with WS are less aroused when presented with negatively valenced social images.

We did not, however, find any differences between the WS participants and the comparison groups in their pupil responses to the nonsocial images. All three groups did not show much arousal to these images, relative to baseline (see Fig. 1). This finding contrasts with those reported by Meyer-Lindenberg et al. (2005) in their fMRI study of high-functioning adults with WS. Specifically, when shown nonsocial scenes (selected from the same collection as our stimuli), high-functioning adults with WS showed increased amygdala activation relative to matched controls. One difference in our studies is in the selection of participants with WS. We included a relatively large number of adolescents and adults whose IQ scores ranged from moderate intellectual disability to low normal. In 
contrast, Meyer-Lindenberg and colleagues only included a small number of adults whose IQ scores were within the normal range. Since our WS group only differed significantly in their pupil response from the CA comparison group on the social images, we cannot rule out that, in this study, the arousal responses from the WS participants might be partially related to their lower MA. It might also be that we had selected less threatening nonsocial stimuli from the IAPS collection than did Meyer-Lindenberg and his colleagues or that amygdala activation is a more sensitive measure of response to threat in nonsocial images than pupil dilation.

In contrast to the viewing time and pupil dilation measures, the likeability ratings collected from our participants are explicit measures that reflect more deliberate reasoning and evaluation of social and nonsocial images. Here, we found that the WS group rated both the social and nonsocial images as either more likable in the case of the positive images or less likeable in the case of the negative images than either comparison group. We had predicted the higher ratings for the social images, but not for the nonsocial images. This pattern of more extreme ratings across all stimuli seems to reflect a more "dramatic" cognitive style that fits with the exuberant personality profile that has been described in the WS literature (e.g., Dykens and Rosner 1999; Gosch and Pankau 1997; KleinTasman and Mervis 2003). Interestingly, however, the negative images, both social and nonsocial, were rated as more negative by the WS participants compared to both age- and MA-matched controls. This finding contrasts with the approachability and trustworthiness ratings reported by Bellugi et al. (1999), who found that adults with WS rated both positive and negative faces as more approachable (positive), but may be more consistent with later studies using this same task, which found that WS participants only gave more extreme ratings for happy (i.e., positive) faces (Frigerio et al. 2006; Porter et al. 2007).

The cross-measure analyses were also quite revealing. Autonomic arousal, reflected in the pupil dilation measure, did not correlate with either viewing time or ratings for any group. This is not surprising given that the latter measures reflect more complex higher-order attentional and cognitive processes, mediated by cortical systems, whereas autonomic arousal is mediated more by lower-level subcortical systems, including the amygdala. For the typically developing children and adults, who were divided into age and MA comparison groups, viewing time and likeability ratings were significantly correlated across different categories of images. Thus, these participants were consistent in that they looked longer at images they liked more and less at images they disliked. For the WS group, this relationship between looking time and ratings did not hold, suggesting that, for this population, there is a disconnection between implicit (looking time) and explicit (ratings) measures of social information processing. In our view, these measures tap into distinct components of the social phenotype of adolescents and adults with WS: looking time reflects their strong attentional interest in social scenes, while likeability ratings reflect their exuberant personality style. People who have interacted with individuals with WS, as well as parents, teachers, and clinicians, often remark on how they show intense emotions, a tendency toward exaggerations and even a demonstrative style in social interactions (Gosch and Pankau 1997; Semel and Rosner 2003), consistent with temperamental exuberance. This style might have been indeed reflected in their explicit appraisal of emotionally laden images with social or nonsocial content. It is important to consider how this disconnect between implicit and explicit processing of social information might impact the quality of the real-life social interactions of people with WS. An apparent lack of awareness of their attention deployment behavior, coupled with decreased arousal in social situations, might lead to interactions that could be perceived as intrusive by other people and lacking expected social inhibitions. Basic attentional and physiological mechanisms like those tapped by our experimental measures can provide clues to why individuals with WS encounter difficulties in their social interactions, despite their overt friendliness and social interest. In our study, the comparison groups were both of typically developing individuals, and we realize that the inclusion of an MAmatched group does not fully control for the potential impact of intellectual disability on performance on the study measures or rule out the possibility that the disconnect between implicit and explicit affective appraisal of social and nonsocial information might be found in other populations with neurodevelopmental disorders. It would be useful to include such contrast groups in future research on WS, using multiple measures for assessing affective appraisal and its relationships to intellectual functioning.

Using a range of measures to investigate how people with WS respond to complex scenes provided a rich view into the way they process emotionally valenced social and nonsocial information, with each measure highlighting distinctive components of the WS phenotype. Complex scenes may be more useful for investigating social phenotypes than relying just on single faces, and recent work on other neurodevelopmental disorders, particularly autism spectrum disorders (ASD), has been expanding the scope of stimuli used to probe social impairments (e.g., Norbury et al. 2009; Sasson et al. 2008; Shic et al. 2011). Future studies of WS should consider investigating responses to complex dynamic as well as static images, using implicit and explicit behavioral measures, psycho- 
physiology, as well as neuroimaging methods, and to compare them not just to typical controls, but to groups such as ASD that are also defined on the basis of a unique social phenotype. Combining rich phenotypic characterization using experimental methods with genetic information studies of these populations can advance our understanding of the genetic bases of social behavior. Finally, the origins and developmental trajectory of attentional processes, psychophysiological reactivity to social information, and exuberant personality style should also be the focus of future research on this unique neurodevelopmental disorder.

Acknowledgements This research was supported by grants from the National Institute of Child Health and Human Development (RO1 HD 33470) and by the CTSA Grant Number 1UL1RR025771 from the National Center for Research Resources (NCRR), a component of the National Institute of Health (NIH). Its contents are solely the responsibility of the authors and do not necessarily represent the official view of NCRR or NIH. We express our sincere thanks to the National Williams Syndrome Association for their help in recruiting participants and to the families and individuals who participated in this study. We gratefully acknowledge Nicole Crawford and Meaghan Kennedy for their help with experiment design and development, Lan Nguyen for her efforts in testing participants, and Alex Storer for his help with data processing and analysis.

\section{References}

Adolphs R. The neurobiology of social cognition. Current Opinion in Neurobiology. 2001;11:231-9.

Adolphs R, Tranel D, Damasio AR. The human amygdala in social judgment. Nature. 1998;393:470-5.

Bellugi U, Wang P, Jernigan TL. Williams syndrome: an unusual neuropsychological profile. In: Broman SH, Grafman J, editors. Atypical cognitive deficits in developmental disorders. Hillsdale: Erlbaum; 1994. p. 23-56.

Bellugi U, Adolphs R, Cassady C, Chiles M. Towards the neural basis for hypersociability in a genetic syndrome. Neuroreport. 1999;10:1653-7.

Dodd HF, Porter MA. I see happy people: attention towards happy but not angry facial expressions in Williams syndrome. Cognitive Neuropsychiatry. 2010;15:549-67.

Doyle TF, Bellugi U, Korenberg JR, Graham J. "Everybody in the world is my friend": hypersociability in young children with Williams syndrome. American Journal of Medical Genetics. 2004;124A:263-73.

Dykens EM, Rosner B. Refining behavioral phenotypes: personalitymotivation in Williams and Prader-Willi syndromes. American Journal on Mental Retardation. 1999;104:158-69.

Ewart AK, Morris CA, Atkinson D, Jin W, Sternes K, Spallone P, Stock AD, Leppert M, Keating MT. Hemizygosity at the elastin locus in a developmental disorder, Williams syndrome. Nature Genetics. 1993;5:11-6.

Frigerio E, Burt DM, Gagliardi C, Cioffi G, Martelli S, Perrett DI, Borgatti R. Is everybody always my friend? Perception of approachability in Williams syndrome. Neuropsychologia. 2006;44:254-9.

Gagliardi C, Frigerio E, Burt DM, Cazzaniga I, Perrett D, Borgatti R. Facial expression recognition in Williams syndrome. Neuropsychologia. 2003;41:733-8.
Gosch A, Pankau R. Personality characteristics and behavior problems in individuals of different ages with Williams syndrome. Developmental Medicine and Child Neurology. 1997;39:327-533.

Granholm E, Steinhauer SR. Pupillometric measures of cognitive and emotional processes. International Journal of Psychophysiology. 2004;52:1-6.

Haas B, Mills D, Yam A, Hoeft F, Bellugi U, Reiss A. Genetic influences on sociability: heightened amygdala reactivity and event-related responses to positive social stimuli in Williams syndrome. Journal of Neuroscience. 2009;29:1132-9.

Hess EH. Pupillometrics: a method of studying mental, emotional, and sensory processes. In: Greenfield NS, Sternbach RA, editors. Handbook of psychophysiology. New York: Holt, Rinehart \& Winston; 1972. p. 491-531.

Howlin P, Udwin O. Outcome in adult life for people with Williams syndrome-results from a survey of 239 families. Journal of Intellectual Disability Research. 2006;50:151-60.

Janisse MP. Pupil size and affect: a critical review of the literature since 1960. Canadian Psychologist. 1973;14:311-29.

John AE, Mervis CB. Comprehension of the communicative intent behind pointing and gazing gestures by young children with Williams syndrome or Down syndrome. Journal of Speech, Language, and Hearing Research. 2010;53:950-60.

Jones W, Bellugi U, Lai Z, Chiles M, Reilly J, Lincoln A, Adolphs R. Hypersociability in Williams syndrome. Journal of Cognitive Neuroscience. 2000;12(Supplement):30-46.

Kasthurirangan S, Glasser A. Age related changes in the characteristics of the near pupil response. Vision Research. 2006;46:1393-403.

Kaufman A, Kaufman N. Manual for the Kaufman Brief Test of Intelligence. 2nd ed. Circle Pines: American Guidance Service; 2004.

Klein-Tasman BP, Mervis CB. Distinctive personality characteristics of 8-, 9-, and 10-year-olds with Williams syndrome. Developmental Neuropsychology. 2003;23:269-90.

Korenberg JR, Chen XN, Hirota H, Lai Z, Bellugi U, Burian D, Roe B, Matsuoka R. Genome structure and cognitive map of Williams syndrome. Journal of Cognitive Neuroscience. 2000;12(Suppl):89 107.

Lang PJ, Bradley MM, Cuthbert BN. International affective picture system (IAPS): affective ratings of pictures and instruction manual. Technical report A-8. Gainesville: University of Florida; 2008.

LeDoux JE. Emotion circuits in the brain. Annual Review of Neuroscience. 2000;23:155-84.

Libby Jr WL, Lacey BC, Lacey JI. Pupillary and cardiac activity during visual attention. Psychophysiology. 1973;10:270-94.

Lowenfeld IE. Pupillary changes related to age. In: Thompson SH, editor. Topics in neuro-ophtalmology. Woburn: ButterworthHeinemann; 1979. p. 83-272.

Martens MA, Wilson SJ, Dudgeon P, Reutens DC. Approachability and the amygdala: insights from Williams syndrome. Neuropsychologia. 2009;47:2446-53.

Mervis CB, Robinson BF, Bertrand J, Morris CA, Klein-Tasman BP, Armstrong SC. The Williams syndrome cognitive profile. Brain and Cognition. 2000;44:604-28.

Mervis C, Morris CA, Klein-Tasman BP, Bertrand J, Kwitny S, Appelbaum LG, Rice CE. Attentional characteristics of infants and toddlers with Williams syndrome during triadic interactions. Developmental Neuropsychology. 2003;2:243-68.

Meyer-Lindenberg A, Hariri AR, Munoz KE, Mervis CB, Mattay VS, Morris CA, Berman KF. Neural correlates of genetically abnormal social cognition in Williams syndrome. Nature Neuroscience. 2005;8:991-3.

Meyer-Lindenberg A, Mervis CB, Berman KF. Neural mechanisms in Williams syndrome: a unique window to genetic influences on cognition and behavior. Nature Reviews: Neuroscience. 2006;7:380 93. 
Mobbs D, Garrett AS, Menon V, Rose FE, Bellugi U, Reiss AL. Anomalous brain activation during face and gaze processing in Williams syndrome. Neurology. 2004;6:2070-6.

Morris C, Dilts C, Demsey S, Leonard C, Blackburn B. The natural history of Williams syndrome: physical characteristics. Journal of Pediatrics. 1988;11:318-26.

Muñoz KE, Meyer-Lindenberg A, Hariri AR, Mervis CB, Mattay VS, Morris CA, Berman KF. Abnormalities in neural processing of emotional stimuli in Williams syndrome vary according to social vs. non-social content. Neuroimage. 2010;50(1):340-6.

Norbury CF, Brock J, Cragg L, Einav S, Nation K. Eye-movement patterns are associated with communicative competence in autistic spectrum disorders. Journal of Child Psychology and Psychiatry. 2009;50:834-42.

Paul BM, Snyder AZ, Haist F, Raichle ME, Bellugi U, Stiles J. Amygdala response to faces parallels social behavior in Williams syndrome. Social Cognitive and Affective Neuroscience. 2009;4:27885.

Plesa Skwerer D, Sullivan K, Joffre K, Tager-Flusberg H. Self concept in people with Williams syndrome and Prader-Willi syndrome. Research in Developmental Disabilities. 2004;2:119-38.

Plesa Skwerer D, Faja S, Schofield C, Verbalis A, Tager-Flusberg H. Perceiving facial and vocal expression of emotion in Williams syndrome. American Journal on Mental Retardation. 2006a;111:1526.

Plesa Skwerer D, Verbalis A, Schofield C, Faja S, Tager-Flusberg H. Social-perceptual abilities in adolescents and adults with Williams syndrome. Cognitive Neuropsychology. 2006b;22:1-12.

Plesa Skwerer D, Borum L, Verbalis A, Schofield C, Crawford N, Ciciolla L, Tager-Flusberg H. Autonomic responses to dynamic displays of facial expressions in adolescents and adults with Williams syndrome. Social Cognitive and Affective Neuroscience. 2009;4:93-100.
Porter M, Coltheart M, Langdon R. The neuropsychological basis of hypersociability in Williams and Down syndrome. Neuropsychologia. 2007;45:2839-49.

Porter MA, Coltheart M, Langdon R. Theory of mind in Williams syndrome assessed using a nonverbal task. Journal of Autism and Developmental Disorders. 2008;38:806-14.

Riby DM, Hancock PJ. Viewing it differently: social scene perception in Williams syndrome and autism. Neuropsychologia. 2008;46:2855-60.

Riby DM, Hancock PJ. Do faces capture the attention of individuals with Williams syndrome or autism? Journal of Autism and Developmental Disorders. 2009;39:421-31.

Rose FE, Lincoln AJ, Lai Z, Ene M, Searcy YM, Bellugi U. Orientation and affective expression effects on face recognition in Williams syndrome and autism. Journal of Autism and Developmental Disorders. 2007;37:513-22.

Sasson NJ, Turner-Brown LM, Holtzclaw TN, Lam KS, Bodfish JW. Children with autism demonstrate circumscribed attention during passive viewing of complex social and nonsocial picture arrays. Autism Research. 2008;1:31-42.

Semel E, Rosner S. Understanding Williams syndrome. Hillsdale: Lawrence Erlbaum Associates; 2003.

Shic F, Bradshaw J, Klin A, Scassellati B, Chawarska K. Limited activity monitoring in toddlers with autism spectrum disorder. Brain Research. 2011;1380:246-54.

Stinton C, Elison S, Howlin P. Mental health problems in adults with Williams syndrome. American Journal on Intellectual and Developmental Disabilities. 2010;115:3-18.

Tager-Flusberg H, Sullivan K. A componential view of theory of mind: evidence from Williams syndrome. Cognition. 2000;76:59 89.

Tager-Flusberg H, Plesa-Skwerer D, Faja S, Joseph RM. People with Williams syndrome process faces holistically. Cognition. 2003;89:1124. 